\title{
PERSPECTIVAS PARA EL DESARROLLO DE LA FACULTAD DE
}

Por: Hipolito Camacho Coy Decano de la Facultad de Educacion Iniversidad Surcolomhiana

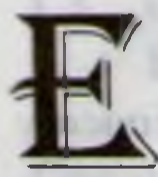
I consejo de facultad de educación con el fin de aglutinar el trabajo de todos los programas en torno a unas perspectivas comunes, estructura para el año 1996 un plan de acción básico, que recoge en gran medida las políticas institucionales.

Este plan, no solo iluminará las acciones de los diferentes organismos de dirección de la facultad, sino que se constituye en un elemento para la evaluación, en la medida que permitirá determinar periódicamente si las metas previstas se estan logrando y en consecuencia, trazar los correctivos del caso.

Tal como lo preveen los criterios modernos de planeación, no constituye un documento rigido y acabado, por el contrario, debe ser tomado como un punto de partida que requiere ser enriquecido a partir de los debates que se generen en los consejos de departamento, en las reuniones de comités, y en las discusiones de los equipos de trabajo que se están consolidando en la facultad.

La incorporación Se procura que luego de las discusiones y ajustes, sea de procesos de asumido como un compromiso colectivo de tal manera que autoevaluación

permanente permitirán mejorar las metas iniciales. toda la comunidad educativa se convierta en protagonista de su desarrollo. La incorporación de procesos de autoevaluación permanente permitirán mejorar las metas iniciales en procura de la maxima calidad en la gestion de directivos, docentes y estudiantes.

El plan presenta de manera suscinta, desde los componentes: Administrativo, de investigación, de extensión y desde los programas académicos diversas opciones de trabajo a saber. 


\section{DESDE EL COMPONENTE ADMINISTRATIVO}

\subsection{APROBACION E IMPLEMENTACION DE LA NUEVA ESTRUCTURA ORGANICA DE LA FACULTAD dE EDUCACIÓN}

Coordinación: Docano de la facultad de educación.

Unidad de Apoyo: Consejo de Facultad.

METAS

- A junio de 1996, aprobación de la nueva estructura en el Consejo Académico y en el Consejo Superior.

- A noviembre de 1996: En funcionamiento los nuevos departamentos. el Instituto de Investigación y el Centro de Proyección Comunitaria.

\section{ACCIONES}

- Presentación y sustentación de la nueva estructura ante el Consejo Académico

- Presentación y sustentación de la nueva estructura ante el Consejo Superior

- Promulgación de los acuerdos respectivos.

- Apropiación de los recursos básicos.

- Designación del personal requerido para su funcionamiento.

\subsection{CREACION Y PUESTA EN MARCHA DEL INSTITUTO DE INVESTIGACIONES}

Coordinación: Coordinador del Comité de Investigaciones de la Facultad. Unidad de Apoyo: Comité de Investigaciones

\section{METAS}

- A junio de 1996: Definido el marco legal, manual de funciones y normatividad para su implementación. Aprobación en el Consejo de Facultad.

- A noviembre de 1996: Definido y asignado el coordinador del Instituto. consolidado el equipo de trabajo y los proyectos básicos para el año 1997.

\section{ACCIONES}

- Definición de la estructura orgánica para el Instituto. 
- Aprobación de la estructura en las diferentes instancias académicas de la Universidad (C. de Facultad, C. Académico y C. Superior).

- Divulgación de las normas y procedimientos.

- Estudio y aprobación de proyectos.

- Negociación de proyectos con entidades del orden internacional, nacional y regional, en procura de cofinanciación.

\subsection{CREACION Y PUESTA EN MARCHA DEL CENTRO DE PROYECCION Y PARTICIPACION COMUNITARIA.}

Coordinación: Coordinador del Comité de Extensión de La Facultad.

Unidad de Apoyo: Comité de Extensión de La Facultad de Educación.

\section{METAS}

- A junio de 1996: Definido el marco legal, manual de funcionamiento, y normatividad para su implementación. Aprobación en las diferentes instancias académicas de la universidad.

- A noviembre de 1996: Definido y asignado el Director del Centro, consolidado el equipo de trabajo y los proyectos para desarrollar en 1997.

\section{ACCIONES}

- Definición y aprobación de la estructura orgánica para el instituto.

- Divulgación de las normas y procesos para su funcionamiento.

- Estudio y aprobación de proyectos, según líneas de acción definidas previamente.

- Negociación de proyectos con entidades de carácter internacional, nacional. regional y local; en procura de su financiación.

- Inscripción de proyectos generales en el Banco Nacional de Proyectos y en las instancias de carácter departamental y municipal.

\subsection{PLAN DE DESARROLLO PARA LA FACULTAD DE EDUCACION 1997-2001}

Coordinación: Coordinador del Comité del Plan de Desarrollo.

Unidad de Apoyo: Comité del Plan de desarrollo. 


\section{METAS}

- A junio de 1996: Desarrolladas las dos primeras etapas, del plan a saber: Diseño del proyecto, autoevaluación de la Facultad.

- A noviembre de 1996: Desarrolladas la tercera y cuarta etapas, a saber: Prospección para el período 1997-2001 y Formulación del Plan.

\section{ACCIONES}

- Cunsulidación de la Teleología para la facultad de Educación . socialización y discusión de un documento básico sobre el particular.

- Elaboración del anteproyecto, presentación, discusión y reestructuración del mismo en el comité ampliado.

- Elaboración y adopción del proyecto.

- Identificación de necesidades del desarrollo que debe atender la facultad. descripción y evaluación de las realizaciones académicas con énfasis en el período 1991-1995.

- Elaboración de la visión de la facultad, elaboración y selección de las alternativas de desarrollo.

- Redefinición de políticas, formulación de los planes de investigación, docencia y extensión, formulación de proyectos.

\subsection{DEFINICION DE POLITICAS Y ESTRATEGIAS PARA LOS CURSOS $Y$ EVENTOS DE CAPACITACION SABATICOS $Y$ COMISIONES DE ESTUDIO}

Coordinación: Secretaría Académica de la Facultad.

Unidad de Apoyo: Consejo de Facultad de Educación.

\section{METAS}

- A junio de 1996: Discutido y consertado un documento base que permita al consejo de facultad tomar decisiones acertadamente en torno a la capacitación de los docentes.

- A noviembre de 1996: Aprobadas las políticas y los mecanismos en las diferentes instancias académicas de la Universidad.

\section{ACCIONES}

- Elaboración de un documento preliminar para la discusión. 
- Análisis amplio del documento, consertación de mecanismos.

- Presentación y aprobación en las diferentes instancias académicas de la Universidad.

- Implementación de los acuerdos para todos los profesores que lo requieran a partir del primer semestre de 1997.

\section{DESDE EL COMPONENTE DE INVESTIGACION}

\subsection{DEFINICION DE LINEAS DE INVESTIGACION PARA LA FACULTAD}

Coordinación: Coordinador Comité de Investigaciones.

Unidad de Apoyo: Comité de Investigaciones y Consejo de Facultad.

\section{METAS}

- A junio de 1996: Definidas las líneas que orientarán la investigación en los próximos 5 años en la Facultad de Educación.

- A noviembre de 1996: Aprobadas las líneas de investigación en las diferentes instancias académicas de la Universidad. Aprobados los proyectos para 1997 a partir de estos criterios.

\section{ACCIONES}

- Reuniones de discusión, concertación y definición de políticas.

- Análisis de las políticas de investigación de COLCIENCIAS, ICFES, FES, CEPECS, UNIVERSIDADES ESTATALES, CESU, y otras entidades que desarrollen y financien investigaciones.

- Articulacion de políticas externas con las políticas internas de la Facultad.

- Aprobación Institucional de las políticas definidas.

\subsection{COFINANCIACION DE PROYECTOS DE INVESTIGACION}

Coordinación: Coordinador del Comité de Investigaciones-Investigadores-Unidad de Apoyo: Instituto de Investigaciones de la Facultad. CIDEC. 


\section{METAS}

- A noviembre de 1996: lograr la cofinanciación de un número no inferior a A...nt... progrootos on la Fasultad du Educación.

\section{ACCIONES}

- Estructuración de Proyectos por parte de los profesores investigadores teniendo en cuenta las líneas definidas por la Universidad y los requerimientos técnicos y académicos de entidades financiadoras.

- Presentación de proyectos a nivel institucional para su aprobación y respaldo institucional.

- Presentación de Investigaciones con respaldo institucional a las entidades financiadoras.

- Seguimiento a los proyectos presentados con miras a obtener respuestas favorables.

\subsection{PUBLICACION DE RESULTADOS}

Coordinación: Director del Instituto de Investigaciones-Investigadores.

Unidad de Apoyo: Instituto de Investigaciones de la facultad.

\section{METAS}

- A noviembre de 1996: Lograr la publicación de dos boletines informativos a nivel interno. Publicación de artículos sobre resultados de las investigaciones en revistas de circulación nacional.

\section{ACCIONES}

- Gestión para financiación de los boletines informativos.

- Presentación de artículos sobre resultados finales y parciales de las investigaciones.

- Gestión ante las revistas especializadas de carácter nacional e internacional.

- Incorporación de un rubro para publicaciones en todas las investigaciones de la Facultad de Educación. 


\section{DESDE EL COMPONENTE DE EXTENSION}

\subsection{CONSOLIDACION DEL SISTEMA DE FORMACION PERMANENTE PARA LOS DOCENTES DEL DEPARTAMENTO DEL HUILA}

Coordinación: Coordinador del Comité de extensión.

Unidad de Apoyo: Comité de Extensión.

\section{METAS}

- A junio de 1996: Presentar el estudio sobre las necesidades de capacitación para los docentes del Huila, a nivel pedagógico y por áreas del conocimiento. Estructuración de diferentes ofertas a nivel local y regional.

- A noviembre de 1996: Tener estructuradas y concertadas con entidades financiadoras, diferentes ofertas para los docentes del Huila. Haber iniciado la implementación de algunas de ellas.

\section{ACCIONES}

- Desarrollo de estudios sobre necesidades de capacitación docente a nivel local y regional.

- Definición de las políticas de capacitación y proyectos específicos.

- Presentación de los proyectos a la Secretaría de Educación Departamental a la Secretaría de Educación de Neiva, y a demás entidades interesadas en el tema.

- Proceso de divulgación de los proyectos de capacitación.

- Puesta en marcha de los proyectos aprobados.

- Seguimiento y evaluación permanente a los proyectos en desarrollo.

\subsection{CONSOLIDACION DE LA OFERTA SOBRE CURSOS LIBRES}

Coordinación: Coordinador Comité de Extensión.

Unidad de Apoyo: Comité de extensión de la facultad.

\section{METAS}

- A junio de 1996: Establecer un sistema para el ofrecimiento de cursos libres, para garantizar la unidad de esfuerzos y lograr máxima eficiencia. 
- A noviembre de 1996: Tener en funcionamiento varias ofertas de cursos libres. adicionales a las que ya existen Tener información centralizada sobre el desarrollo de los mismos.

\section{ACCIONES}

- Hacer un análisis situacional de los cursos libres que hoy se ofrecen en los programas de Lenguas modernas. Artes visuales y el LIFE entre otros.

- Estructurar diferentes ofertas por departamentos y por programas.

- Consolidar una oferta integral de cursos libres que pueda ser ofrecida a unerentes personas naturales o jurídicas.

- Establecimiento de convenios interinstitucionales para el ofrecimiento de cursos a diferentes empresas.

- Definición Financiera y presupuestal.

\subsection{DEFINICION DE CONVENIOS}

Coordinación: Coordinador del Comité de Extensión - Decano.

Unidad de Apoyo: Comité de Investigaciones-Consejo de Facultad.

\section{METAS}

- A noviembre de 1996: Tener firmados varios convenios para capacitación docente y educación comunitaria con el municipio de Neiva. la Secretaría Departamental de Educación, El Ministerio de Educación Nacional. Tener varios proyectos inscritos en los "BANCOS DE PROYECTOS"

\section{ACCIONES}

- Contactos permanentes con las entidades interesadas en la capacitación docente y la educación comunitaria.

- Formulación de proyectos según los requisitos académicos y técnicos de las entidades financiadoras.

- Presentación y sustentación de proyectos a nivel institucional y a nivel externo.

- Seguimiento permanente a los proyectos presentados para financiación externa.

- Seguimiento a la firma de los convenios.

- Implementación de proyectos que logren financiación para 1996.

- Evaluación de proyectos para asegurar apoyo institucional. 


\section{DESDE LOS PROGRAMAS ACADEMICOS Y DEPARTAMENTOS}

\subsection{ACREDITACION DE LOS PROGRAMAS EXISTENTES EN PREGRADO Y POSTGRADO}

Coordinación: Jefes de programas y coordinadores de los postgrados respectivos.

Unidad de Apoyo: Consejos de Programa o Departamento.

\section{METAS}

- A noviembre de 1996: Haber desarrollado el proceso de autoevaluación y adelantando los primeros contactos oficiales para la acreditación de los programas que a partir de los procesos de autoevaluación consideran que llenan los requisitos para tal fin.

\section{ACCIONES}

- Elaboración del proyecto de acreditación a partir de las propuestas elaboradas en 1995.

- Definición de la estrategia metodológica.

- Diseño y aplicación de instrumentos.

- Desarrollo de talleres y confrontación de resultados.

- Elaboración de informe sobre resultado de la autoevaluación.

- Oficialización del proceso ante el Consejo Nacional de acreditación, definición de pares académicos.

- Análisis de las políticas del CESU sobre el particular.

\subsection{CONSOLIDACION DE NUEVAS OFERTAS EN PREGRADO Y POSTGRADO}

Coordinación: Jefes de Programa y Departamento. Coordinadores de Postgrado. Unidad de Apoyo: Consejos de Programa y Departamento, Consejo de Facultad.

\section{METAS}

- A noviembre de 1996: Tener en funcionamiento la Licenciatura de Biología y Quimica, aprobada la Licenciatura en Informática Educativa, estructurada unas propuestas para especialización en el campo de la Lingüistica y Literatura 


\subsection{OFERTAS DE PREGRADO Y POSTGRADO EN LAS SEDES}

Coordinación: Jefes de Programa y Departamentos, Coordinadores de Postgrados. Unidad de Apoyo: Consejos de Programa, Consejos de Departamento, Consejo de Facultad.

\subsection{INTEGRACION DE PROGRAMAS DE PREGRADO Y POSTGRADO}

Coordinación: Jefes de Programa y Departamento, Coordinadores de Postgrado. Unidad de Apoyo: Consejos de Programa, Consejos de Departamento, Consejo de Facultad.

\subsection{REESTRUCTURACION CURRICULAR DE LOS PROGRAMAS DE LA FACULTAD EN CONCORDANCIA CON LAS POLITICAS DE LA LEY 115 Y LA REESTRUCTURACION DE LAS ESCUELAS NORMALES}

Coordinación: Jefes de Programa y Departamento.

Unidad de Apoyo: Consejo de Facultad, Equipo interinstitucional para la reestructuración de las normales en el departamento del Huila. 